\title{
Effects of blood triglycerides on cardiovascular and all-cause mortality: a systematic review and meta-analysis of 61 prospective studies
}

\author{
Jun Liu ${ }^{1 \dagger}$, Fang-Fang Zeng ${ }^{1 \dagger}$, Zhao-Min Liu², Cai-Xia Zhang ${ }^{1}$, Wen-hua Ling ${ }^{1}$ and Yu-Ming Chen ${ }^{1 *}$
}

\begin{abstract}
The relationship of triglycerides (TG) to the risk of death remains uncertain. The aim of this study was to determine the associations between blood triglyceride levels and cardiovascular diseases (CVDs) mortality and all-cause mortality. Four databases were searched without language restriction for relevant studies: PubMed, ScienceDirect, EMBASE, and Google Scholar. All prospective cohort studies reporting an association between TG and CVDs or all-cause mortality published before July 2013 were included. Risk ratios (RRs) with 95\% confidence intervals (Cls) were extracted and pooled according to TG categories, unit TG, and logarithm of TG using a random-effects model with inverse-variance weighting. We identified 61 eligible studies, containing 17,018 CVDs deaths in 726,030 participants and 58,419 all-cause deaths in 330,566 participants. Twelve and fourteen studies, respectively, reported the effects estimates of CVDs and total mortality by TG categories. Compared to the referent $(90-149 \mathrm{mg} / \mathrm{dL})$, the pooled RRs ( $95 \% \mathrm{Cl}$ ) of CVDs mortality for the lowest (< $90 \mathrm{mg} / \mathrm{dL}$ ), borderline-high (150-199 mg/dL), and high TG ( $\geq 200 \mathrm{mg} / \mathrm{dL}$ ) groups were 0.83 (0.75 to 0.93$), 1.15$ (1.03 to 1.29), and 1.25 (1.05 to 1.50); for total mortality they were 0.94 (0.85 to 1.03), 1.09 (1.02 to 1.17), and 1.20 (1.04 to 1.38), respectively. The risks of CVDs and all-cause deaths were increased by $13 \%$ and $12 \%(p<0.001)$ per 1 -mmol/L TG increment in twenty-two and twenty-two studies reported RRs per unit TG, respectively. In conclusion, elevated blood TG levels were dose-dependently associated with higher risks of CVDs and all-cause mortality.
\end{abstract}

Keywords: Triglycerides, Cardiovascular disease, All-cause, Mortality, Meta-analysis

\section{Introduction}

There is considerable evidence to suggest that triglycerides (TG) play a role in such adverse health conditions as heart disease, peripheral vascular disease, stroke, diabetes mellitus, metabolic syndrome, and cancer, which are common causes of death [1-3]. Several reviews and meta-analyses evaluated the association between blood TG and mortality. Hokanson et al. reported the summary crude RR for and CVDs death in the sensitive analysis of a meta-analysis assessing the association of TG with CVDs incidence [4], in which seventeen prospective studies of American and European participants were included. Two meta-analyses by Hokanson et al. updated

\footnotetext{
* Correspondence: chenyum@mail.sysu.edu.cn

${ }^{\dagger}$ Equal contributors

'Guangdong Provincial Key Laboratory of Food, Nutrition, and Health, School of Public Health, Sun Yat-sen University, Guangzhou, Guangdong 510080,

People's Republic of China

Full list of author information is available at the end of the article
}

the overall effect of TG on CVDs incidence but not for CVDs mortality $[5,6]$. The collaboration studies from the Asia Pacific Cohort Studies Collaboration (APCSC) suggested that elevated TG levels were strongly associated with an increased mortality of coronary heart disease (CHD) $[7,8]$. Another collaborative analysis including 10,269 participants from 7 studies in Europe, reported that higher triglyceride $(\geq 1.7$ versus. $<1.7 \mathrm{mmol} / \mathrm{L}$ ) was associated with an increased risk of CVDs (but not all-cause) moratity in women and men [9]. Up to date, no study has assessed the overall effects of TG on CVDs death or all-cause death by including all eligible studies in the world. We conducted a systematic review and meta-analysis of prospective studies to evaluate the association between blood TG levels and CVDs mortality and all-cause mortality.

\section{Biomed Central}

(c) 2013 Liu et al.; licensee BioMed Central Ltd. This is an open access article distributed under the terms of the Creative Commons Attribution License (http://creativecommons.org/licenses/by/2.0), which permits unrestricted use, distribution, and reproduction in any medium, provided the original work is properly cited. 


\section{Methods}

\section{Literature search strategies}

Following the methodology advocated in the Meta-Analysis of Observational Studies in Epidemiology (MOOSE) guidelines [10] and without language restrictions, we carried out a systematic search of four electronic databases, PubMed (1966 to July 2013), ScienceDirect (1960 to July 2013), EMBASE (1980 to July 2013), and Google Scholar (http:// scholar.google.com) without language restrictions using the following search terms: (i) triglyceride* or triacylglycerol* or TG or lipids; (ii) blood or serum or plasma or circulating; (iii) cohort* or longitudinal or follow-up or prospective or relative risk* or hazard ratio*; (iv) death* or mortali*; and (v) (i) AND (ii) AND (iii) AND (iv). We also searched the reference lists of original and review articles to identify more studies. Related studies generated by PubMed were also retrieved. If necessary, we contacted authors for additional data.

\section{Inclusion and exclusion criteria}

We included studies that met all of the following criteria: (1) prospective cohort design in a general population; (2) the exposure of interest included TG; (3) the outcome of interest included all-cause mortality or CVDs mortality; and (4) the relative risks (RRs) or hazard ratios (HRs) with their corresponding 95\% confidence intervals (CIs) (or sufficient data to estimate them) were reported according to at least three TG categories, or by unit of TG, or by logarithmically transformed TG. We excluded studies that were focused on patients with one or several specific types of disease, such as diabetes, CVDs, dyslipidemia, or cancer. If multiple published reports from the same population resource or overlapping datasets were available, we included only the most relevant article with the largest dataset.

\section{Data extraction and quality assessment}

Two epidemiologists (JL and FFZ) independently assessed the eligible studies, collected information, and assessed the quality of the data. Any discrepancies were resolved through discussion. All relevant information was recorded, included the article title, the first author's name, publication year, country in which the study was performed, population inclusion and exclusion criteria, sample size at baseline, mean (range) duration of follow-up, mean (range) age at baseline, proportion of men, geographic location, type of blood sample (serum, plasma, or other), fasting status (yes, no, or other), whether there was an adjustment for total cholesterol (TC) or not, and risk estimate (RR or HR) with corresponding 95\% CI after controlling for both the minimally and the maximally adjusted number of covariates.

The Newcastle-Ottawa Quality Assessment Scale (NOS) was applied to assess the quality of each included study [11]. The NOS was designed to assesses the quality of cohort studies in terms of their selection of participants (four criteria), degree of comparability between study groups (one criterion), and assessment of outcomes (three criteria). Total scores range from 1 to 9 , with 9 being the maximum.

\section{Statistical analysis}

We conducted three separate meta-analyses for the risk estimates on the basis of the TG categories, unit TG, and logarithm of TG. For the category data, we standardized and grouped the TG levels with unit $\mathrm{mg} / \mathrm{dL}$ into the four categories used in the Adult Treatment Panel III (ATP III) cholesterol guidelines [12]: lowest group $(<90 \mathrm{mg} / \mathrm{dL})$, intermediate group ( $90-149 \mathrm{mg} / \mathrm{dL}$ ), borderline hypertriglyceridemia group (150-199 mg/dL), and hypertriglyceridemia group $(\geq 200 \mathrm{mg} / \mathrm{dL}$ ). The intermediate group was used as the referent. As the highest category in each individual study was open-ended, we assigned the category a value equal to $50 \%$ of the width of its lower boundary. If one or more group in a single study fell into the same category, we pooled these risk estimates first. We analyzed the dose-response relationships using the method proposed by Greenland et al. [13,14]. Study-specific slopes (linear trends) were calculated from the natural log of the RRs across various exposure levels, correlated with their corresponding TG levels. The original dose groups were used in the dose-response relationship analysis. For the studies reporting the risk estimates by unit TG, the RRs were standardized to a $1-\mathrm{mmol} / \mathrm{L}$ increase in TG according to the following formulas.

$$
\begin{aligned}
R R_{\mathrm{st}} & =\exp \left(\ln R R_{o} / k\right) \\
95 \% \mathrm{CI} & =\exp \left(\ln R R_{\mathrm{o}} / k \pm 1.96 S E_{\mathrm{o}} / k\right) \\
S E_{\mathrm{o}} & =\left(\ln R R_{\mathrm{o} \_ \text {ul }}-\ln R R_{\mathrm{o} \_l l}\right) /(2 * 1.92)
\end{aligned}
$$

Where, $R R_{s t}$ is the standardized RR; $R R_{o}$ is derived from the confidence intervals provided in each study; $u l$ is the upper limit; $l l$ is the lower limit; and $k$ refers to the per $k$-mmol/L increase in TG levels in the original study.

For the studies reporting the risk estimates by logarithmically transformed TG, we pooled the risk estimates per unit of log of TG (mmol/L).

The DerSimonian and Laird random-effects model of inverse variance methods was used to estimate the pooled risks and 95\% CIs [15]. RRs were used for all of the risk estimates including RRs and HRs. Unless otherwise stated, we used the most fully adjusted RRs from each study. Statistical heterogeneity was tested with the $I^{2}$ statistic; $I^{2}$ values of $25 \%$ to $<50 \%, 50 \%$ to $<75 \%$, and $\geq 75 \%$ were considered to represent small, medium, and large degree of inconsistency, respectively [16]. The Begg's and Egger's regression tests were used to detect any publication bias 


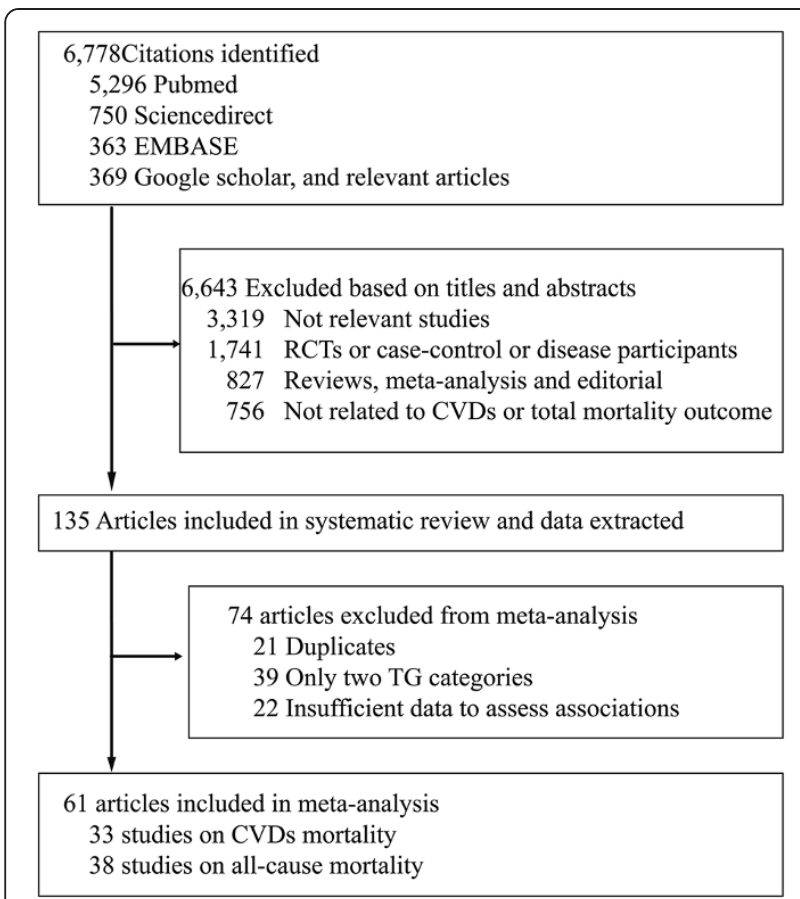

Figure 1 Flow diagram of study selection. TG: Triglycerides; CVDs: Cardiovascular diseases.

$[17,18]$ and a trim-and-fill method was used to adjust for any suspected publication bias once identified [19].

Subgroup analyses were carried out to identify possible sources of heterogeneity and to check for the potential effects of age ( $\geq 50$ or not), gender (men/women), duration of follow-up ( $\geq 15$ or not), sample size ( $\geq 4000$ or not), fasting status (Yes or No), geographic location (Europe/America or Asia-Pacific), blood sample (serum/ plasma), free CVDs history at baseline (Yes or No), study quality ( $>6$ score or not), and adjustment for TC (Yes or No) on the relationship between TG and the outcomes. We also performed a meta-regression to explore the independent influences of these factors on heterogeneity and then sensitivity analysis on the main effects by excluding all of the studies one by one to evaluate whether any single or group of studies might markedly affect the results. We also compared the pooled estimates using RRs adjusted for the minimal and maximal number of covariates in the studies that reported both. Finally, using the methods proposed by Mantel and Haenszel, we performed the chi-square test for heterogeneity to examine differences in pooled RRs [20]. All of these tests were two-sided and statistical significance was defined as $P<0.05$. The analyses were performed with the Stata statistical software version 11.0 (College Station, TX, USA).

\section{Results}

\section{Study characteristics}

Figure 1 outlines our search and selection process, and illustrates the detailed population selection criteria in the original studies. A total of 61 studies included in the present meta-analysis were pooled according to CVDs mortality and all-cause mortality. They were all

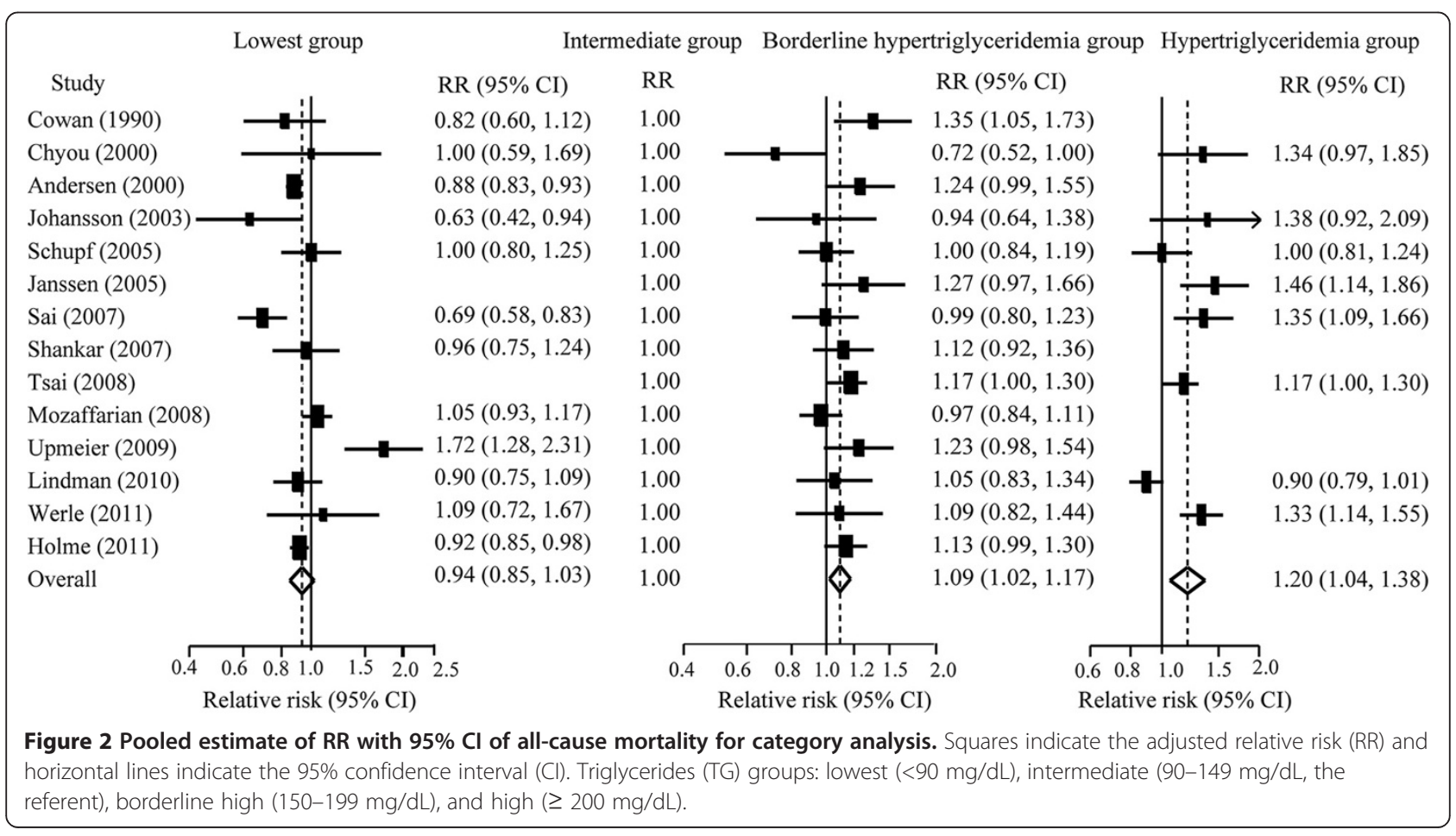




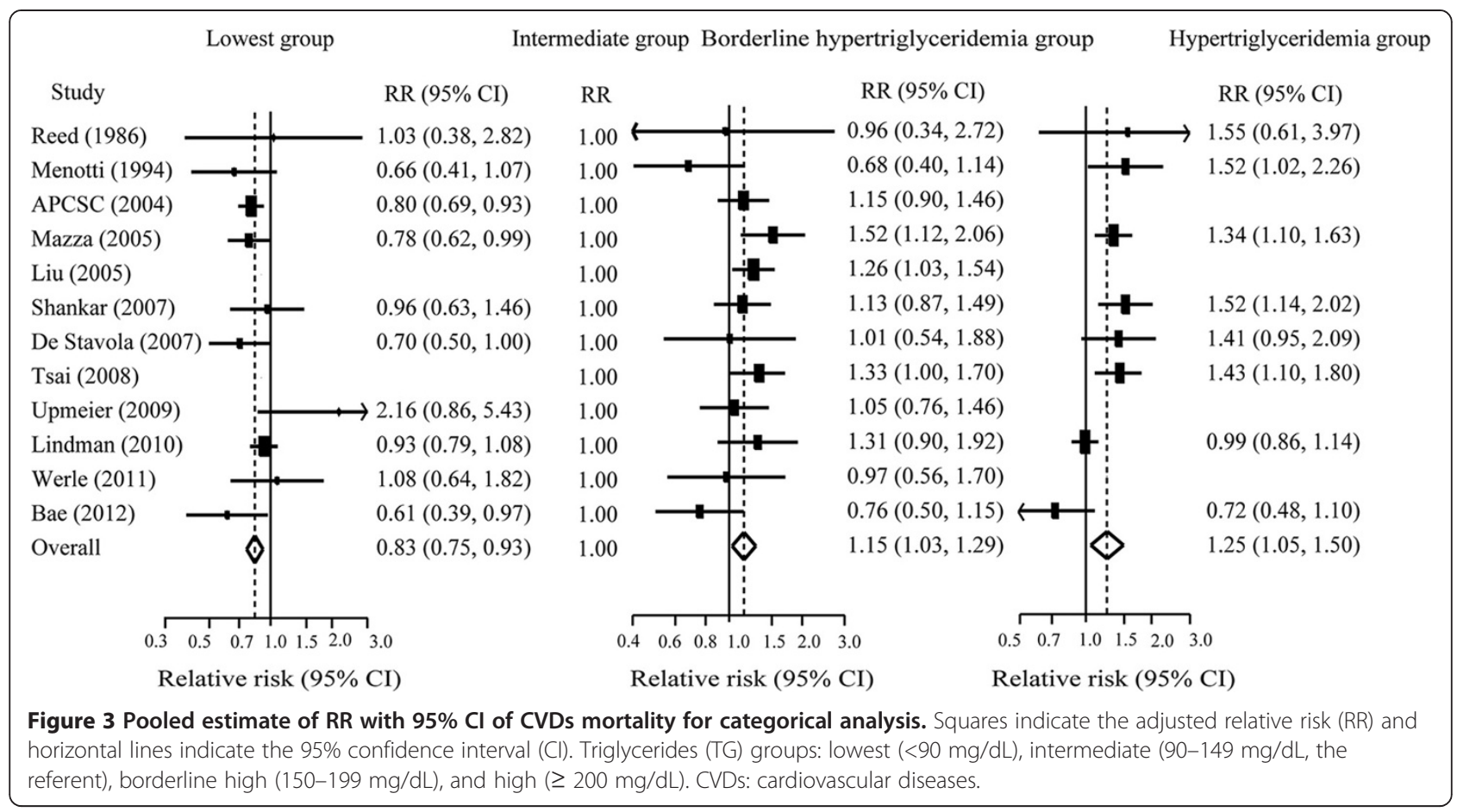

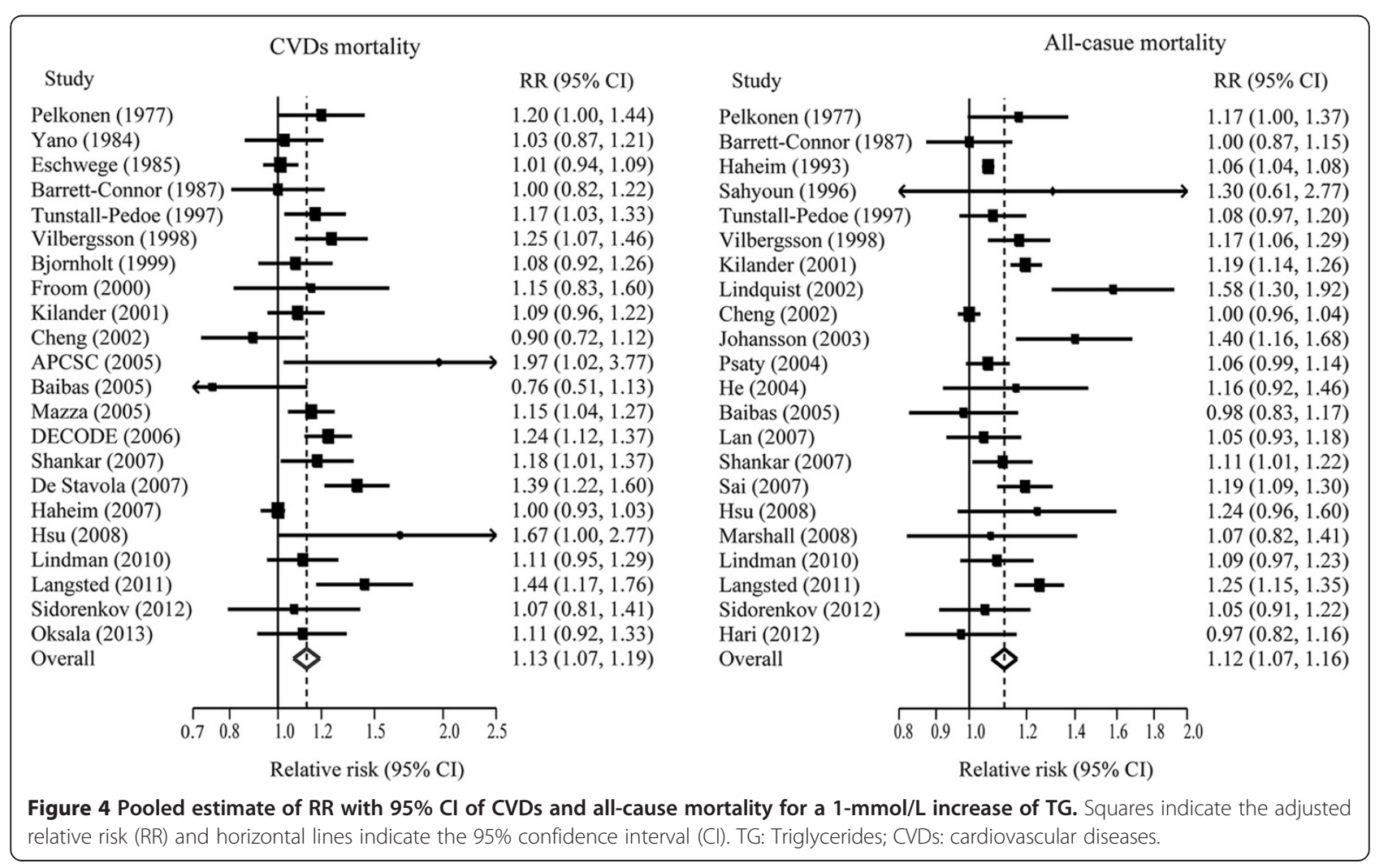




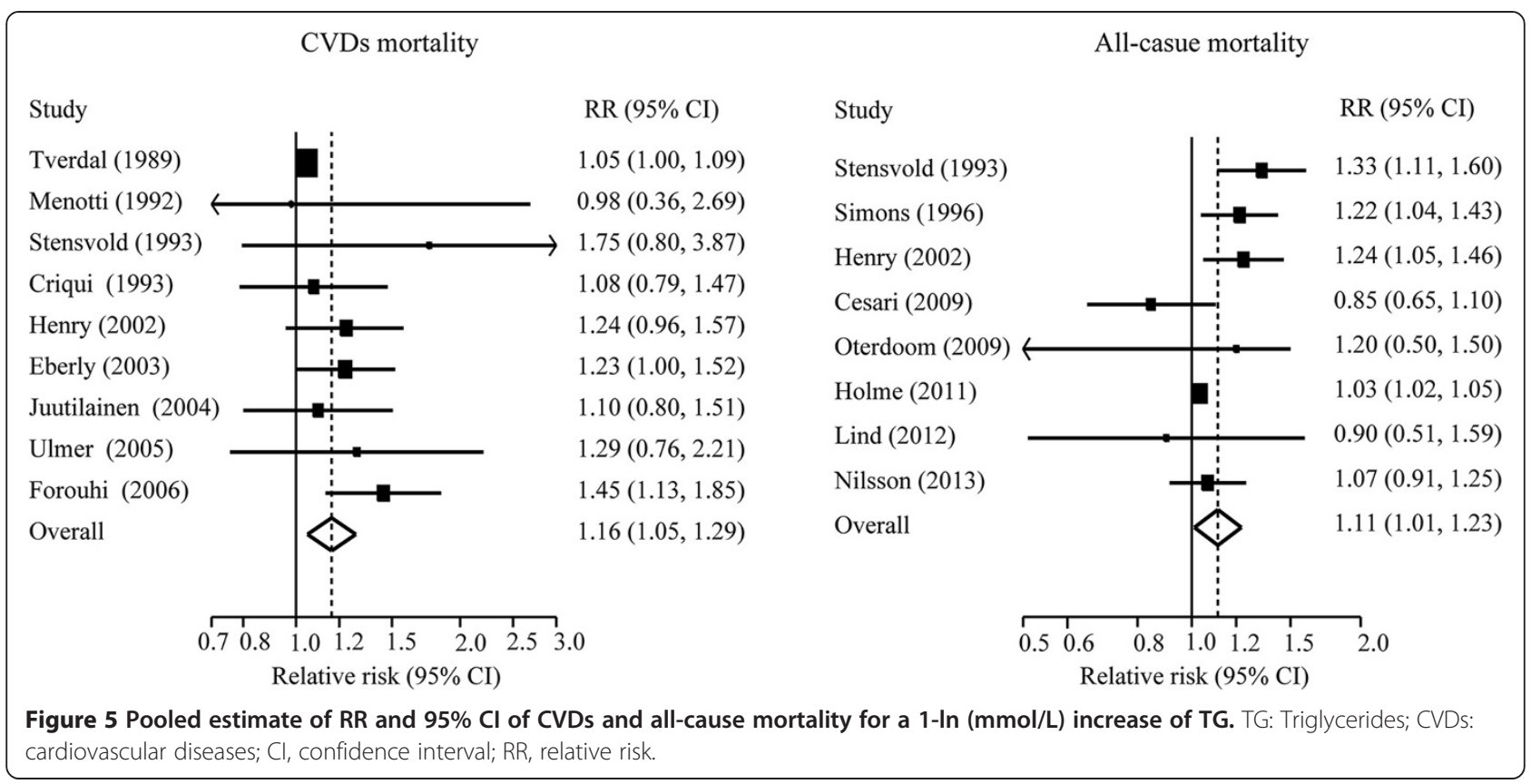

published in English with the exception of one published in Norwegian study [21]. The features of these studies are summarized in Additional file 1: Table S1. Thirtythree studies reported data on CVDs mortality; together recording 17,018 CVDs deaths in 726,030 participants; and 38 studies reported data on all-cause mortality, together recording 58,419 deaths in 330,566 participants.
The median age at the recruitment was 48.0 and median duration of follow-up was 12.0 years.

\section{Overall effects of triglycerides on CVDs and all-cause mortality}

Twelve and fourteen studies, respectively, reported the effects estimates of CVDs and total mortality by TG

Table 1 Pooled relative risks of CVDs and all-cause mortality by blood TG levels

\begin{tabular}{|c|c|c|c|c|c|c|c|c|}
\hline & \multirow[t]{2}{*}{$\mathbf{N}$} & \multicolumn{2}{|l|}{ Overall effects } & \multirow{2}{*}{$\begin{array}{l}I^{2} \\
(\%)\end{array}$} & \multirow{2}{*}{$\begin{array}{l}P \text { for } \\
\text { heterogeneity }\end{array}$} & \multirow{2}{*}{$\begin{array}{l}\text { Begg's test } \\
\text { (P value) }\end{array}$} & \multicolumn{2}{|c|}{ Influence analyses } \\
\hline & & $\mathrm{RR}_{(95 \% \mathrm{Cls})}{ }^{\dagger}$ & $P$ & & & & Minimum & Maximum \\
\hline \multicolumn{9}{|c|}{ Cardiovascular disease mortality } \\
\hline \multicolumn{9}{|l|}{ TG groups, mg/dl } \\
\hline$<90$ & 10 & $0.83(0.75,0.93)$ & 0.001 & 21.6 & 0.244 & 0.592 & $0.80(0.71,0.91)$ & $0.85(0.76,0.94)$ \\
\hline $90-150$ & 12 & 1.00 (referent) & & & & & & \\
\hline $150-200$ & 12 & $1.15(1.03,1.29)$ & 0.015 & 22.5 & 0.222 & 0.150 & $1.12(1.00,1.27)$ & $1.19(1.08,1.32)$ \\
\hline$>200$ & 8 & $1.25(1.05,1.50)$ & 0.013 & 66.2 & 0.004 & 0.711 & $1.22(1.00,1.49)$ & $1.33(1.13,1.57)$ \\
\hline Per $1 \mathrm{mmol} / \mathrm{L}$ TG & 22 & $1.13(1.07,1.19)$ & 0.000 & 65.9 & 0.000 & 1.000 & $1.11(1.06,1.17)$ & $1.14(1.08,1.20)$ \\
\hline Per $1 \ln (\mathrm{mmol} / \mathrm{L} \mathrm{TG})$ & 9 & $1.16(1.05,1.29)$ & 0.004 & 32.3 & 0.160 & 0.754 & $1.06(1.02,1.11)$ & $1.24(1.11,1.39)$ \\
\hline \multicolumn{9}{|l|}{ All-cause mortality } \\
\hline \multicolumn{9}{|l|}{ TG groups, mg/dl } \\
\hline$<90$ & 12 & $0.94(0.85,1.03)$ & 0.150 & 71.1 & 0.000 & 0.732 & $0.90(0.83,0.97)$ & $0.96(0.84,1.06)$ \\
\hline $90-150$ & 14 & 1.00 (referent) & & & & & & \\
\hline $150-200$ & 14 & $1.09(1.02,1.17)$ & 0.011 & 33.3 & 0.108 & 1.000 & $1.08(1.00,1.16)$ & $1.11(1.04,1.19)$ \\
\hline $00 \mathrm{~A} 0>200$ & 8 & $1.20(1.04,1.38)$ & 0.011 & 75.4 & 0.000 & 0.711 & $1.17(1.01,1.35)$ & $1.25(1.14,1.37)$ \\
\hline Per $1 \mathrm{mmol} / \mathrm{L}$ TG & 22 & $1.12(1.07,1.16)$ & 0.000 & 75.9 & 0.000 & 0.866 & $1.11(1.07,1.15)$ & $1.13(1.08,1.18)$ \\
\hline Per 1 In (mmol/L TG) & 8 & $1.11(1.01,1.23)$ & 0.030 & 62.4 & 0.010 & 0.711 & $1.08(0.99,1.18)$ & $1.14(1.02,1.28)$ \\
\hline
\end{tabular}

† Random-effects model.

$\mathrm{RR}$, relative risk; $\mathrm{Cl}$, confidence interval; TG: triglycerides; CVDs: cardiovascular diseases. 
Table 2 Pooled relative risks of CVDs mortality for categorical and continuous analyses in subgroups

\begin{tabular}{|c|c|c|c|c|c|c|c|c|c|c|}
\hline \multirow[t]{3}{*}{ Sub-groups } & \multicolumn{8}{|c|}{ Triglyceride group (mg/dl) } & \multirow{2}{*}{\multicolumn{2}{|c|}{ Per $1 \mathrm{mmol} / \mathrm{L}$ TG }} \\
\hline & \multicolumn{2}{|r|}{$<90$} & \multicolumn{2}{|c|}{$90-146$} & \multicolumn{2}{|r|}{$150-199$} & \multicolumn{2}{|r|}{$\geq \mathbf{2 0 0}$} & & \\
\hline & $\mathrm{N}^{*}$ & $\mathrm{RR}(95 \% \mathrm{Cl})$ & $\mathrm{N}^{*}$ & RRt & $\mathrm{N}^{*}$ & RR (95\% Cl) & $\mathbf{N}^{*}$ & $\mathrm{RR}(95 \% \mathrm{Cl})$ & $\mathrm{N}^{*}$ & RR $(95 \% \mathrm{Cl})$ \\
\hline \multicolumn{11}{|l|}{ Age (year) } \\
\hline$<50$ & 3 & $0.84(0.74,0.96)$ & 4 & 1.00 & 4 & $1.21(1.06,1.40)$ & 3 & $1.20(0.94,1.53)$ & 10 & $1.11(1.01,1.22)$ \\
\hline$\geq 50$ & 7 & $0.85(0.68,1.05)$ & 8 & 1.00 & 8 & $1.08(0.90,1.30)$ & 5 & $1.30(0.99,1.69)$ & 12 & $1.15(1.09,1.22)$ \\
\hline$P$ value & & 0.956 & & & & 0.338 & & 0.664 & & 0.488 \\
\hline \multicolumn{11}{|l|}{ Gender } \\
\hline Male & 5 & $0.86(0.67,1.09)$ & 5 & 1.00 & 5 & $1.06(0.89,1.30)$ & 5 & $1.31(1.12,1.15)$ & 18 & $1.10(1.04,1.15)$ \\
\hline Female & 3 & $0.76(0.64,0.90)$ & 3 & 1.00 & 3 & $1.37(1.09,1.71)$ & 2 & $2.08(1.44,3.00)$ & 9 & $1.40(1.22,1.59)$ \\
\hline Mixed & 4 & $1.01(0.65,1.56)$ & 6 & 1.00 & 6 & $1.14(1.10,1.31)$ & 4 & $1.26(0.99,1.60)$ & 4 & $1.16(1.08,1.25)$ \\
\hline$P$ value & & 0.416 & & & & 0.100 & & 0.023 & & 0.001 \\
\hline
\end{tabular}

Follow-up (year)

\begin{tabular}{|c|c|c|c|c|c|c|c|c|c|c|}
\hline$<12$ & 6 & $0.81(0.71,0.91)$ & 6 & 1.00 & 6 & $1.01(0.84,1.19)$ & 4 & $1.25(0.84,1.84)$ & 11 & $1.14(1.06,1.22)$ \\
\hline$\geq 12$ & 4 & $0.79(0.61,1.02)$ & 4 & 1.00 & 6 & $1.27(1.13,1.44)$ & 4 & $1.25(1.01,1.54)$ & 11 & $1.11(1.02,1.21)$ \\
\hline$P$ value & & 0.473 & & & & 0.025 & & 0.992 & & 0.694 \\
\hline
\end{tabular}

Fast status

\begin{tabular}{|c|c|c|c|c|c|c|c|c|c|c|}
\hline Fast & 6 & $0.89(0.71,1.12)$ & 7 & 1.00 & 7 & $1.15(0.98,1.35)$ & 4 & $1.42(1.22,1.64)$ & 10 & $1.10(1.04,1.17)$ \\
\hline Nonfast & 3 & $1.00(0.65,1.54)$ & 4 & 1.00 & 4 & $1.14(0.85,1.46)$ & 4 & $1.11(0.85,1.46)$ & 7 & $1.12(1.01,1.23)$ \\
\hline Others $^{\S}$ & 1 & $0.80(0.69,0.93)$ & 1 & 1.00 & 1 & $1.15(0.90,1.45)$ & 0 & - & 5 & $1.19(1.00,1.41)$ \\
\hline$P$ value & & 0.646 & & & & 0.843 & & 0.122 & & 0.821 \\
\hline
\end{tabular}

Geographic location

\begin{tabular}{|c|c|c|c|c|c|c|c|c|c|c|}
\hline Europe/America & 7 & $0.86(0.73,1.01)$ & 8 & 1.00 & 8 & $1.17(1.00,1.37)$ & 5 & $1.22(1.02,1.55)$ & 18 & $1.11(1.05,1.17)$ \\
\hline Asia-Pacific & 3 & $0.80(0.70,0.92)$ & 4 & 1.00 & 4 & $1.20(0.93,1.35)$ & 3 & $1.20(0.82,1.77)$ & 4 & $1.26(1.05,1.52)$ \\
\hline$P$ value & & 0.509 & & & & 0.729 & & 0.847 & & 0.205 \\
\hline
\end{tabular}

Quality score

\begin{tabular}{|c|c|c|c|c|c|c|c|c|c|c|}
\hline$<7$ & 4 & $0.76(0.65,0.90)$ & 5 & 1.00 & 5 & $1.01(0.80,1.29)$ & 4 & $1.24(0.91,1.68)$ & 8 & $1.16(1.04,1.29)$ \\
\hline 7-9 & 6 & $0.92(0.77,1.05)$ & 7 & 1.00 & 7 & $1.23(1.09,1.38)$ & 4 & $1.25(0.98,1.60)$ & 14 & $1.11(1.04,1.19)$ \\
\hline$P$ value & & 0.100 & & & & 0.167 & & 0.961 & & 0.509 \\
\hline
\end{tabular}

Sample size

$\begin{array}{lllllllllll}<4000 & 7 & 0.84(0.70,1.02) & 7 & 1.00 & 7 & 1.10(0.91,1.32) & 4 & 1.49(1.21,1.80) & 10 & 1.14(1.06,1.22) \\ \geq 4000 & 3 & 0.83(0.71,0.97) & 5 & 1.00 & 5 & 1.18(1.02,1.38) & 4 & 1.12(0.84,1.45) & 12 & 1.13(1.04,1.22) \\ P \text { value } & & & & & & & & 0.510 & & \end{array}$

Free of CVDs at baseline

\begin{tabular}{|c|c|c|c|c|c|c|c|c|c|c|}
\hline Yes & 6 & $0.85(0.76,0.96)$ & 7 & 1.00 & 7 & $1.12(0.92,1.36)$ & 6 & $1.20(0.96,1.50)$ & 13 & $1.11(1.04,1.20)$ \\
\hline No & 4 & $0.88(0.67,1.56)$ & 5 & 1.00 & 5 & $1.16(1.00,1.34)$ & 2 & $1.43(1.16,1.76)$ & 9 & $1.16(1.09,1.24)$ \\
\hline$P$ value & & 0.832 & & & & 0.811 & & 0.265 & & 0.371 \\
\hline
\end{tabular}

Adjustment

$1.52(1.14,2.02) \quad 7 \quad 1.15(1.08,1.22)$

$\begin{array}{lllllllllll}\text { Minimally } & 4 & 0.72(0.55,0.94) & 5 & 1.00 & 5 & 1.44(1.16,1.78) & 4 & 1.52(1.14,2.02) & 7 & 1.15(1.08,1.22) \\ \text { Maximally } & 4 & 0.87(0.77,0.98) & 5 & 1.00 & 5 & 1.24(1.05,1.46) & 4 & 1.29(1.01,1.65) & 7 & 1.05(0.99,1.11) \\ \text { P value } & & & & & & & & & 0.271 & \end{array}$

Adjustment for TC

\begin{tabular}{|c|c|c|c|c|c|c|c|c|c|c|}
\hline Yes & 4 & $0.85(0.77,0.94)$ & 4 & 1.00 & 4 & $1.24(1.07,1.43)$ & 3 & $1.27(0.94,1.71)$ & 14 & $1.09(1.03,1.16)$ \\
\hline No & 6 & $0.82(0.61,1.09)$ & 8 & 1.00 & 8 & $1.06(0.89,1.26)$ & 5 & $1.25(0.97,1.61)$ & 8 & $1.21(1.10,1.34)$ \\
\hline$P$ value & & 0.810 & & & & 0.169 & & 0.927 & & 0.078 \\
\hline
\end{tabular}


Table 2 Pooled relative risks of CVDs mortality for categorical and continuous analyses in subgroups (Continued)

\begin{tabular}{|c|c|c|c|c|c|c|c|c|c|c|}
\hline \multicolumn{11}{|c|}{ Adjustment for $\mathrm{HDL}$} \\
\hline Yes & 3 & $0.81(0.72,0.91)$ & 3 & 1.00 & 3 & $1.23(1.04,1.47)$ & 1 & $1.52(1.14,2.02)$ & 3 & $1.10(1.03,1.19)$ \\
\hline No & 7 & $0.84(0.68,1.04)$ & 9 & 1.00 & 9 & $1.09(0.94,1.27)$ & 7 & $1.21(1.00,1.47)$ & 19 & $1.13(1.06,1.20)$ \\
\hline$P$ value & & 0.734 & & & & 0.313 & & 0.200 & & 0.679 \\
\hline
\end{tabular}

*The number of cohorts.

†Reference group.

§Others included mixed and unknown ones

$\mathrm{RR}$, relative risk; $\mathrm{Cl}$, confidence interval; CVDs: cardiovascular diseases; HDL: high-density lipoprotein.

TG: triglycerides; TC: total cholesterol.

categories. When compared to individuals with TG level (90 mg/dL-149 mg/dL), those with TG level less than $90 \mathrm{mg} / \mathrm{dL}$ had no significant risk of all-cause mortality, but had less risk of CVDs moratlity, those with borderline hypertriglyceridemia (150 mg/dl-199 mg/dl) hypertriglyceridemia ( $\geq 200 \mathrm{mg} / \mathrm{dL}$ ) had a greater risk of CVDs and all-cause mortality (Figure 2 and Figure 3). The doseresponse analysis showed that the risk of CVDs and total mortality was increased by $13 \%(8 \%-19 \%)$ and $10 \%$ (6\%-14\%) per $1-\mathrm{mmol} / \mathrm{L}$ TG increment (Additional file 2: Figure S2).

Twenty-two and twenty-two studies, respectively, were included in the analysis of risk for CVDs and total mortality by unit TG, and nine and eight studies, respectively, reported the results of CVDs and total mortality by log TG. Figure 4 and Figure 5 show overall association of TG with the risks of CVDs and all-cause mortality.

The heterogeneity across studies varied from null to large $\left(I^{2}=0 \%-75.9 \%\right)$ and no obvious publication biases were observed ( $P=0.150$ to 1.00$)$ in the foregoing analyses (Table 1).

\section{Sensitivity and subgroup analyses}

The subgroup analyses showed that the pooled RRs were significantly higher in men than in women and higher in studies with longer follow-up durations for the risk of both CVDs and total mortality; and tended to be higher for CVDs mortality in the studies that adjusted for TC (RR: 1.09 versus $1.21, P=0.078$ ). Similar results were observed in the meta-regression analyses. No other significant between-group heterogeneities were observed in the subgroup analyses (Tables 2 and 3) or in the metaregression (Additional file 3: Table S3).

Sensitivity and influence analyses were conducted on the pooled risks and the associations of the lowest group tended to be vague when one studies [22] was excluded for total mortality. No single study significantly influenced the pooled estimates risks by unit of TG or for any other of TG categories group. The pooled maximally adjusted RR of CVDs mortality was markedly lower than that of its minimally adjusted $R R$ in the continuous analysis ( $R R=1.05$ versus $1.15, P=0.033)$, but not in the categorical analysis ( $P$ : 0.207 to 0.398). No significant differences were observed in the all-cause mortality data (P: 0.222 to 0.487 ) (Tables 2 and 3 ).

\section{Discussion}

We quantitatively assessed the association between blood triglyceride levels at baseline and CVDs mortality in 33 studies with 17,018 cases among 726,030 participants, as well as all-cause mortality in 38 studies with 58,419 cases among 360,566 participants. Compared to the referent, the risks of CVDs and all-cause mortality were increased by $15.0 \%$ and $9.0 \%$ in the borderline hypertriglyceridemia group, and $25 \%$ and $20 \%$ in the hypertriglyceridemia group, respectively. Overall, the risks of CVDs and all-cause death were increased by $13 \%$ and $12 \%$ per $1-\mathrm{mmol} / \mathrm{L}$ increase in TG level.

These findings were robust. We incorporated a large number of participants and deaths (a total of 17,018 CVDs deaths in 726,030 participants and 58,419 all-cause deaths in 330,566 participants), which improved the statistical power for detecting potential associations. Additionally, the studies included in our sample were carried out worldwide, with participants from the America, Europe, Asia, and Australia, thus enhancing generalizability. Furthermore, only prospective cohort studies were considered. The participants in 33 of the studies had no history of CVDs at baseline and the follow-up duration in 44 was more than 10 years, largely eliminating the possibility of reverse causation relationship. Participants were also selected from the general population, thus reducing potential selection bias. In addition, we used the Newcastle-Ottawa Scale to evaluate the quality of studies and most of the studies (50 studies) included in this meta-analysis were high-quality (score $>6$, full score $=9$ ). Finally, there was a large degree of consistency between the continuous and categorical analyses.

We observed that elevated TG levels were associated with an increased risk of CVDs mortality echoing the findings of several other meta-analyses of prospective studies demonstrating a relationship between higher TG levels and an increased risk of cardiovascular events [4,5,23]. In 1996, Hokanson et al. [4] performed a metaanalysis comprising 46,413 men and 10,864 women from the USA and Europe. Their summary crude RRs for fatal 
Table 3 Pooled relative risks of all-cause mortality for categorical and continuous analyses in subgroups

\begin{tabular}{|c|c|c|c|c|c|c|c|c|c|c|}
\hline \multirow[t]{3}{*}{ Sub-groups } & \multicolumn{8}{|c|}{ Triglyceride group (mg/dl) } & \multirow{2}{*}{\multicolumn{2}{|c|}{ Per $1 \mathrm{mmol} / \mathrm{L} \mathrm{TG}$}} \\
\hline & \multicolumn{2}{|r|}{$<90$} & \multicolumn{2}{|c|}{$90-146$} & \multicolumn{2}{|r|}{$150-199$} & \multicolumn{2}{|r|}{$\geq 200$} & & \\
\hline & $\mathrm{N}^{*}$ & RR (95\% CI) & $\mathrm{N}^{*}$ & RRt & $\mathrm{N}^{*}$ & RR (95\% Cl) & $\mathbf{N}^{*}$ & RR $(95 \% \mathrm{Cl})$ & $\mathrm{N}^{*}$ & RR $(95 \% \mathrm{Cl})$ \\
\hline \multicolumn{11}{|l|}{ Age (year) } \\
\hline$<50$ & 3 & $0.88(0.78,1.00)$ & 5 & 1.00 & 5 & $1.14(1.05,1.24)$ & 5 & $1.20(0.99,1.46)$ & 8 & $1.13(1.05,1.21)$ \\
\hline$\geq 50$ & 9 & $0.97(0.85,1.11)$ & 9 & 1.00 & 9 & $1.07(0.97,1.18)$ & 3 & $1.20(0.97,1.48)$ & 14 & $1.12(1.07,1.17)$ \\
\hline$P$ value & & 0.311 & & & & 0.329 & & 0.982 & & 0.845 \\
\hline \multicolumn{11}{|l|}{ Gender } \\
\hline Male & 8 & $0.95(0.84,1.09)$ & 9 & 1 & 9 & $1.12(1.02,1.22)$ & 5 & $1.23(1.09,1.38)$ & 14 & $1.09(1.04,1.13)$ \\
\hline Female & 6 & $0.91(0.84,0.99)$ & 6 & 1 & 6 & $1.17(0.99,1.39)$ & 2 & $1.01(0.59,1.74)$ & 10 & $1.24(1.15,1.32)$ \\
\hline Mixed & 3 & $1.00(0.85,1.17)$ & 4 & 1 & 4 & $1.11(1.01,1.21)$ & 3 & $1.17(1.01,1.35)$ & 6 & $1.08(1.03,1.13)$ \\
\hline$P$ value & & 0.577 & & & & 0.621 & & 0.491 & & 0.001 \\
\hline \multicolumn{11}{|l|}{ Follow-up (year) } \\
\hline$<15$ & 6 & $0.89(0.84,0.94)$ & 7 & 1.00 & 7 & $1.11(0.97,1.26)$ & 4 & $1.26(1.06,1.51)$ & 14 & $1.05(1.02,1.07)$ \\
\hline$\geq 15$ & 6 & $0.94(0.79,1.11)$ & 7 & 1.00 & 7 & $1.09(1.02,1.16)$ & 4 & $1.15(0.93,1.41)$ & 8 & $1.22(1.16,1.29)$ \\
\hline$P$ value & & 0.569 & & & & 0.773 & & 0.490 & & 0.000 \\
\hline \multicolumn{11}{|l|}{ Fast status } \\
\hline Fast & 8 & $1.01(0.86,1.20)$ & 11 & 1.00 & 11 & $1.08(0.98,1.18)$ & 5 & $1.27(1.09,1.48)$ & 15 & $1.12(1.06,1.19)$ \\
\hline Nonfast & 2 & $0.91(0.86,0.98)$ & 3 & 1.00 & 3 & $1.14(1.04,1.24)$ & 3 & $1.11(0.88,1.40)$ & 6 & $1.11(1.03,1.19)$ \\
\hline Others $^{\S}$ & 2 & $0.79(0.63,0.99)$ & 2 & 1.00 & 2 & $1.11(0.89,1.38)$ & 0 & - & 1 & $1.19(1.09,1.30)$ \\
\hline$P$ value & & 0.258 & & & & 0.381 & & 0.335 & & 0.740 \\
\hline \multicolumn{11}{|l|}{ Geographic location } \\
\hline Europe/America & 10 & $0.96(0.87,1.06)$ & 11 & 1.00 & 11 & $1.09(1.00,1.19)$ & 6 & $1.19(0.98,1.45)$ & 16 & $1.12(1.06,1.17)$ \\
\hline Asia-Pacific & 2 & $0.81(0.59,1.11)$ & 3 & 1.00 & 3 & $1.12(1.02,1.23)$ & 2 & $1.22(1.08,1.39)$ & 6 & $1.13(1.08,1.20)$ \\
\hline$P$ value & & 0.302 & & & & 0.633 & & 0.813 & & 0.640 \\
\hline \multicolumn{11}{|l|}{ Quality score } \\
\hline$<7$ & 5 & $0.90(0.86,0.94)$ & 7 & 1.00 & 7 & $1.15(1.08,1.24)$ & 4 & $1.22(1.07,1.40)$ & 10 & $1.13(1.08,1.18)$ \\
\hline $7-9$ & 7 & $0.95(0.77,1.17)$ & 7 & 1.00 & 7 & $1.03(0.91,1.17)$ & 4 & $1.19(0.91,1.57)$ & 12 & $1.11(1.05,1.18)$ \\
\hline$P$ value & & 0.594 & & & & 0.116 & & 0.869 & & 0.707 \\
\hline \multicolumn{11}{|l|}{ Sample size } \\
\hline$<4000$ & 7 & $0.97(0.75,1.25)$ & 7 & 1.00 & 7 & $1.03(0.92,1.15)$ & 4 & $1.22(1.03,1.45)$ & 13 & $1.14(1.07,1.22)$ \\
\hline$\geq 4000$ & 5 & $0.92(0.86,0.96)$ & 7 & 1.00 & 7 & $1.14(1.04,1.24)$ & 4 & $1.18(0.95,1.45)$ & 9 & $1.09(1.04,1.14)$ \\
\hline$P$ value & & 0.729 & & & & 0.155 & & 0.778 & & 0.190 \\
\hline \multicolumn{11}{|c|}{ Free of CVDs at baseline } \\
\hline Yes & 5 & $0.94(0.85,1.03)$ & 6 & 1.00 & 6 & $1.07(0.99,1.16)$ & 5 & $1.24(0.99,1.56)$ & 13 & $1.10(1.04,1.16)$ \\
\hline No & 7 & $0.97(0.80,1.16)$ & 8 & 1.00 & 8 & $1.10(0.99,1.23)$ & 3 & $1.14(1.00,1.30)$ & 9 & $1.13(1.07,1.20)$ \\
\hline$P$ value & & 0.711 & & & & 0.663 & & 0.519 & & 0.480 \\
\hline \multicolumn{11}{|l|}{ Adjustment } \\
\hline Minimally & 9 & $0.86(0.72,1.03)$ & 10 & 1.00 & 10 & $1.14(0.99,1.30)$ & 7 & $1.38(1.13,1.67)$ & 8 & $1.12(1.01,1.24)$ \\
\hline Maximally & 9 & $0.94(0.80,1.11)$ & 10 & 1.00 & 10 & $1.07(0.99,1.17)$ & 7 & $1.18(1.01,1.38)$ & 8 & $1.07(1.01,1.13)$ \\
\hline$P$ value & & 0.466 & & & & 0.487 & & 0.222 & & 0.432 \\
\hline \multicolumn{11}{|l|}{ Adjustment for TC } \\
\hline Yes & 5 & $0.84(0.7,0.99)$ & 5 & 1.00 & 5 & $1.03(0.93,1.14)$ & 2 & $1.10(0.88,1.38)$ & 11 & $1.10(1.05,1.15)$ \\
\hline No & 7 & $1.00(0.89,1.12)$ & 9 & 1.00 & 9 & $1.12(1.02,1.24)$ & 8 & $1.27(1.16,1.40)$ & 11 & $1.14(1.07,1.21)$ \\
\hline$P$ value & & 0.103 & & & & 0.225 & & 0.239 & & 0.382 \\
\hline
\end{tabular}


Table 3 Pooled relative risks of all-cause mortality for categorical and continuous analyses in subgroups (Continued)

\begin{tabular}{|c|c|c|c|c|c|c|c|c|c|c|}
\hline \multicolumn{11}{|c|}{ Adjustment for $\mathrm{HDL}$} \\
\hline Yes & 2 & $1.03(0.93,1.14)$ & 2 & 1.00 & 2 & $1.02(0.89,1.17)$ & 1 & $1.35(1.09,1.66)$ & 4 & $1.07(1.04,1.11)$ \\
\hline No & 10 & $0.92(0.82,1.02)$ & 12 & 1.00 & 12 & $1.11(1.03,1.20)$ & 8 & $1.14(0.93,1.39)$ & 18 & $1.12(1.07,1.17)$ \\
\hline$P$ value & & 0.115 & & & & 0.296 & & 0.250 & & 0.132 \\
\hline
\end{tabular}

*The number of cohorts.

†Reference group.

§Others included mixed and unknown ones

$\mathrm{RR}$, relative risk; $\mathrm{Cl}$, confidence interval; CVDs: cardiovascular diseases; HDL: high-density lipoprotein.

TG: triglycerides; TC: total cholesterol.

CVDs from seven prospective studies were 1.24 in men and 1.84 in women per a $1-\mathrm{mmol} / \mathrm{L}$ increase. In the present meta-analysis, the RRs of CVDs mortality was lower. However, the discrepancy was unlikely to be caused by geographic variations as we observed similar results in the European and American and Asia-Pacific samples in the present study. Hypertriglyceridemia is commonly associated with diabetes, obesity, hypertension, and smoking, which are independent risk factors for CHD [24,25]. The pooled crude RR in the study by Hokanson et al. might be confounded by these factors as the adjusted overall RR of fatal and non-fatal CVDs for women declined from the crude value of 1.76 to 1.37 [4]. The subgroup analysis of the seven studies that reported both maximally and minimally adjusted data demonstrated that the overall RRs were significantly lower for the maximally adjusted original data, than that for the minimally adjusted. In the present meta-analysis, the data in the majority of the studies were adjusted for common potential confounders, such as age, gender, blood pressure, BMI, diabetes, smoking and TC, and would thus have better validity than unadjusted data.

We also found that an association between elevated TG levels and an increased risk of all-cause mortality. In fact, TG levels were found to have a similar predictive power for CVDs and all-cause mortality. It was thus reasonable to hypothesize that there was a positive relationship between TG and non-CVDs death. Prospective studies have found that elevated TG levels increases the risk of nonCVDs mortality [26]. A collaborative study of metabolic syndrome and cancer (Me-Can) involving 514,097 participants with a 13.4-year follow-up, demonstrated a positive association between serum TG and risk of cancer overall and at several sites. The RR (95\% CI) for the top quintile versus the bottom quintile of triglycerides for overall cancer was 1.16 (1.06 to 1.26 ) in men and 1.15 (1.05 to 1.27 ) in women [3]. Previous studies have also reported that elevated TG levels increased the risks of other deaths, such as from kidney disease [27,28] or suicide [29]. Hence, non-CVDs mortality may contribute to the relative risk of all-cause mortality.

There are several possible explanations for these findings. Firstly, TG is associated with atherogenic remnant particles, which have a high uptake into macrophages leading to foam cell formation. Furthermore, triglyceriderich lipoproteins and their remnants promote inflammation and increase the expression of coagulation factors or leukocyte adhesion molecules [2]. An increased number of atherogenic particles may thus adversely influence CVDs risk. Secondly, hypertriglyceridemia is associated with the development of oxidative stress and reactive oxygen species (ROS) [30]. ROS directly influences cell proliferation and apoptosis and modulates DNA methylation patterns; and thus may contribute to the multistage carcinogenesis process [31]. Additionally, increased oxidative stress in fat has been shown to be an important pathogenic pathway in metabolic syndrome. In addition, elevated triglyceride-rich lipoproteins may adversely influence the risk of chronic kidney diseases, as triglyceride-rich apolipoprotein Bcontaining lipoproteins promote the progression of renal insufficiency [32]. Moreover, oxidative stress and endothelial dysfunction may cause atherosclerosis-related kidney damage in older people [33]. Finally, hypertriglyceridemia has been associated with increased cortisol levels in response to stress and symptoms of depression, which may lead to an increased risk of suicide [29].

In the present study, we found that elevated blood TG was associated with greater total death and CVDs death risk in women than in men, which was consistent with prior studies $[4,5]$. A collaborative study and a large prospective study also showed that TC and HDL-c were more predictive of CVDs mortality in women than in men $[34,35]$. However, the mechanism remains unclear and further studies are needed to clarify this issue. The subgroup analysis in the present study showed that studies with longer follow-up periods had significantly higher risk estimates for mortality, possibly due to the greater cumulative risks associated with longer TG exposure.

This meta-analysis has several limitations. First, there was considerable heterogeneity among the studies included with analysis showing that the major sources of heterogeneity to be gender, adjustment for TC, and follow-up duration. Second, 28 studies that included participants with a prior history of CVDs at baseline were not excluded. Previous studies have reported that excluding participants with a history of CVDs at baseline did not substantially change 
the risks of CVDs mortality [36]. In addition, subgroup analysis suggested that the overall RRs for all-cause and CVDs mortality in relation to TG were not markedly influenced by prior CVDs history. Third, elevated TG levels were accompanied by low HDL-c and high TC, both of which have been associated with a higher CVDs risk [1,2]. In the present meta-analysis, subgroup analysis observed that the effect sizes were significantly decreased after adjustment for TC although the positive relationship remains significant. The overall effect estimates were unlikely to be biased by TC since most of original studies (45/61) reported TC-adjusted RRs, but it tended to be overestimated because few (10/61) studies reported HDL-adjusted RRs. Fourth, the studies included in the present meta-analysis were mainly based on a one-time measurement of baseline blood TG (five studies adjusted for regression dilution bias), which may underestimate the associations due to regression dilution effects. Finally, the relatively small number of studies that included categorical analysis limited the ability to detect heterogeneity in the subgroup analysis.

In conclusion, elevated blood TG levels were dosedependently associated with a greater risk of both CVDs and all-cause mortality. The findings of this metaanalysis suggest that controlling TG can help to prevent CVDs and other causes of death.

\section{Additional files}

Additional file 1: Table S1. Characteristics of the original studies and the study population.

Additional file 2: Figure S1. Dose-response relationships between TG and risk of CVDs and all-cause mortality. The dots represent the RRs corresponding to TG levels in each individual study. The area of the dots is inversely proportional to the logarithm of the RR variance. The three curves are the RR estimates and their 95\% Cls according to the doseresponse model. CVDs, cardiovascular diseases; $\mathrm{Cl}$, confidence interval; RR, relative risk; $\mathrm{TG}$, triglycerides.

Additional file 3: Table S3. Meta regression analysis of TG and CVDs and all-cause mortality for continuous analysis.

\section{Abbreviations}

TG: Triglycerides; CVDs: Cardiovascular diseases; APCSC: Asia pacific cohort studies collaboration; CHD: Coronary heart disease; TC: Total cholesterol; HDL-C: High-density lipoprotein; BMI: Body mass index; ROS: Reactive oxygen species

\section{Competing interests}

The authors declare that they have no competing interests.

\section{Authors' contributions \\ $J \mathrm{~L}$ and FFZ contributed to the collation of data and their critical review, and manuscript writing. JL performed the meta-analysis. YMC concepted and designed the study, and critically revised the paper and had responsibility for its final content. ZML, CXZ and WHL critically revised the paper. All authors have read and approved the final manuscript.}

\section{Acknowledgements}

This study was jointly supported by the 5010 Program for Clinical Researches (No. 2007032) by the Sun Yat-sen University, Guangzhou, P. R. China and the Key Program of National Natural Science Foundation of China (No. 81130052).

\section{Author details}

'Guangdong Provincial Key Laboratory of Food, Nutrition, and Health, School of Public Health, Sun Yat-sen University, Guangzhou, Guangdong 510080, People's Republic of China. ${ }^{2}$ Department of Medicine and Therapeutics, The Chinese University of Hong Kong, Hong Kong, SAR, People's Republic of China.

Received: 27 August 2013 Accepted: 21 October 2013

Published: 29 October 2013

\section{References}

1. Kohli P, Cannon CP: Triglycerides: how much credit do they deserve? Med Clin North Am 2012, 96:39-55.

2. Miller MSN, Ballantyne C, Bittner V, Criqui MH, Ginsberg HN, Goldberg AC, Howard WJ, Jacobson MS, Kris-Etherton PM, Lennie TA, Levi M, Mazzone T, Pennathur S: Triglycerides and cardiovascular disease: a scientific statement from the American Heart Association. Circulation 2011, 123:2292-2333.

3. Borena WST, Jonsson H, Strohmaier S, Nagel G, Bjørge T, Manjer J, Hallmans G, Selmer R, Almquist M, Häggström C, Engeland A, Tretli S, Concin H, Strasak A, Stattin P, Ulmer H: Serum triglycerides and cancer risk in the metabolic syndrome and cancer (Me-Can) collaborative study. Cancer Causes Control 2011, 22:291-299.

4. Hokanson JE, Austin MA: Plasma triglyceride level is a risk factor for cardiovascular disease independent of high-density lipoprotein cholesterol level: a meta-analysis of population-based prospective studies. J Cardiovasc Risk 1996, 3:213-219.

5. Abdel-Maksoud MF, Hokanson JE: The complex role of triglycerides in cardiovascular disease. Semin Vasc Med 2002, 2:325-333.

6. Morrison A, Hokanson JE: The independent relationship between triglycerides and coronary heart disease. Vasc Health Risk Manag 2009, 5:89-95.

7. Collaboration APCS: A comparison of the associations between risk factors and cardiovascular disease in Asia and Australasia. Eur J Cardiovasc Prev Rehabil 2005, 12:484-491.

8. Collaboration APCS: Serum triglycerides as a risk factor for cardiovascular diseases in the Asia-Pacific region. Circulation 2004, 110:2678-2686.

9. The DECODE Study Group: Comparison of different definitions of the metabolic syndrome in relation to cardiovascular mortality in European men and women. Diabetologia 2006, 49:2837-2846.

10. Stroup DF BJ, Morton SC, Olkin I, Williamson GD, Moher D, Becher BJ, Sipe $T A$, Thacker SB: Meta-analysis of observational studies in epidemiology: a proposal for reporting. Meta-analysis Of Observational Studies in Epidemiology (MOOSE) group. JAMA 2000, 283:2008-2012.

11. Wells GSB, O'Connell D: The Newcastle-Ottawa Scale (NOS) for assessing the quality of nonrandomized studies in meta-analyses. http://www.ohri.ca/ programs/clinical_epidemiology/oxford.asp.

12. Expert Panel on Detection: Evaluation, and Treatment of High Blood Cholesterol in Adults: Executive Summary of The Third Report of The National Cholesterol Education Program (NCEP) Expert Panel on Detection, Evaluation, And Treatment of High Blood Cholesterol In Adults (Adult Treatment Panel III). JAMA 2001, 285:2486-2497.

13. Greenland S, Longnecker MP: Methods for trend estimation from summarized dose-response data, with applications to meta-analysis. Am J Epidemiol 1992, 135:1301-1309.

14. Berlin JA, Longnecker MP, Greenland S: Meta-analysis of epidemiologic dose-response data. Epidemiology 1993, 4:218-228.

15. DerSimonian R, Laird N: Meta-analysis in clinical trials. Control Clin Trials 1986, 7:177-188

16. Higgins JP, Thompson SG, Deeks JJ, Altman DG: Measuring inconsistency in meta-analyses. BMJ 2003, 327:557-560

17. Begg $C B$, Mazumdar $M$ : Operating characteristics of a rank correlation test for publication bias. Biometrics 1994, 50:1088-1101.

18. Egger M, Davey Smith G, Schneider M, Minder C: Bias in meta-analysis detected by a simple, graphical test. BMJ 1997, 315:629-634.

19. Duval S, Tweedie R: Trim and fill: a simple funnel-plot-based method of testing and adjusting for publication bias in meta-analysis. Biometrics 2000, 56:455-463.

20. Mantel N, Haenszel W: Statistical aspects of the analysis of data from retrospective studies of disease. J Natl Cancer Inst 1959, 22:719-748. 
21. Holme I, Tonstad S: Risk factors and mortality-a follow-up of the Oslo Health Study from 1972-73. Tidsskr Nor Laegeforen 2011, 131:456-460.

22. Upmeier E, Lavonius S, Lehtonen A, Viitanen M, Isoaho H, Arve S: Serum lipids and their association with mortality in the elderly: a prospective cohort study. Aging Clin Exp Res 2009, 21:424-430.

23. Sarwar NDJ, Eiriksdottir G, Sigurdsson G, Wareham N, Bingham S, Boekholdt SM, Khaw KT, Gudnason V: Triglycerides and the risk of coronary heart disease: 10,158 incident cases among 262,525 participants in 29 Western prospective studies. Circulation 2007, 115:450-458.

24. Hulley SB, Rosenman RH, Bawol RD, Brand RJ: Epidemiology as a guide to clinical decisions. The association between triglyceride and coronary heart disease. N Engl J Med 1980, 302:1383-1389.

25. Grundy SM: Hypertriglyceridemia, atherogenic dyslipidemia, and the metabolic syndrome. Am J Cardiol 1998, 81:18B-25B.

26. Langsted A, Freiberg JJ, Tybjaerg-Hansen A, Schnohr P, Jensen GB, Nordestgaard BG: Nonfasting cholesterol and triglycerides and association with risk of myocardial infarction and total mortality: the Copenhagen City Heart Study with 31 years of follow-up. J Intern Med 2011, 270:65-75.

27. Navaneethan SD, Schold JD, Arrigain S, Thomas G, Jolly SE, Poggio ED, Schreiber MJ Jr, Sarnak MJ, Nally JV Jr: Serum triglycerides and risk for death in Stage 3 and Stage 4 chronic kidney disease. Nephrol Dial Transplant 2012, 27:3228-3234.

28. Thomas G, Sehgal AR, Kashyap SR, Srinivas TR, Kirwan JP, Navaneethan SD: Metabolic syndrome and kidney disease: a systematic review and metaanalysis. Clin J Am Soc Nephrol 2011, 6:2364-2373.

29. Chang SS, Wen CP, Tsai MK, Lawlor DA, Yang YC, Gunnell D: Adiposity, its related biologic risk factors, and suicide: a cohort study of 542,088 taiwanese adults. Am J Epidemiol 2012, 175:804-815.

30. Cowey S, Hardy RW: The metabolic syndrome: A high-risk state for cancer? Am J Pathol 2006, 169:1505-1522

31. Ishino K, Mutoh M, Totsuka Y, Nakagama H: Metabolic syndrome: a novel high-risk state for colorectal cancer. Cancer Lett 2013, 33:56-31. doi:10.1016/j.canlet.2012.10.012. Epub ahead of print.

32. Samuelsson O, Attman PO, Knight-Gibson C, Larsson R, Mulec H, Weiss L, Alaupovic P: Complex apolipoprotein B-containing lipoprotein particles are associated with a higher rate of progression of human chronic renal insufficiency. J Am Soc Nephrol 1998, 9:1482-1488.

33. Cheng HT, Huang JW, Chiang CK, Yen CJ, Hung KY, Wu KD: Metabolic syndrome and insulin resistance as risk factors for development of chronic kidney disease and rapid decline in renal function in elderly. J Clin Endocrinol Metab 2012, 97:1268-1276.

34. Lewington S, Whitlock G, Clarke R, Sherliker P, Emberson J, Halsey J, Qizilbash N, Peto R, Collins R: Blood cholesterol and vascular mortality by age, sex, and blood pressure: a meta-analysis of individual data from 61 prospective studies with 55,000 vascular deaths. Lancet 2007, 370:1829-1839.

35. Stensvold I, Urdal P, Thurmer H, Tverdal A, Lund-Larsen PG, Foss OP: Highdensity lipoprotein cholesterol and coronary, cardiovascular and all cause mortality among middle-aged Norwegian men and women. Eur Heart J 1992, 13:1155-1163

36. Mazza A, Tikhonoff V, Schiavon L, Casiglia E: Triglycerides + high-densitylipoprotein-cholesterol dyslipidaemia, a coronary risk factor in elderly women: the CArdiovascular STudy in the ELderly. Intern Med J 2005, 35:604-610

Cite this article as: Liu et al.: Effects of blood triglycerides on cardiovascular and all-cause mortality: a systematic review and metaanalysis of 61 prospective studies. Lipids in Health and Disease 2013 12:159.

\section{Submit your next manuscript to BioMed Central and take full advantage of:}

- Convenient online submission

- Thorough peer review

- No space constraints or color figure charges

- Immediate publication on acceptance

- Inclusion in PubMed, CAS, Scopus and Google Scholar

- Research which is freely available for redistribution 